\title{
Identification of Deceptive Reviews by Sentimental Analysis and Characteristics of Reviewers
}

\author{
Xinyu Gao ${ }^{1}$, Shi $\mathrm{Li}^{1{ }^{1 * *}}$, Yiyin $\mathrm{Zhu}^{1}$, Yiting $\mathrm{Nan}^{2}$, Zihan Jian ${ }^{1}$ and Hongyu Tang ${ }^{1}$ \\ ${ }^{1}$ College of Information and Computer Engineering, Northeast Forestry University, Harbin 150000, China \\ ${ }^{2}$ Inode Ink Corporation Broomfield, CO 80021 , United States
}

Received 20 December 2018; Accepted 26 February 2019

\begin{abstract}
The purchase decisions of customers are influenced by the relevant reviews made by customers. Deceptive reviews are confusing and hard to detect. The existing identification method of deceptive reviews is based on the traditional algorithm of machine learning. However, the methods used to identify deceptive reviews must be improved. In order to improve the accuracy of the identification of deceptive reviews, a novel method integrating sentimental analysis and the characteristics of reviewers was proposed in this study. On the basis of the analysis of the emotional characteristics of the review texts and the behavioural characteristics of the reviewers, a method of deceptive review identification was established. The proposed method analyzed the intensity of emotions, the text similarity, the largest daily publishing comment index, and the extreme rating index on the basis of the feature-weighted model. This model verified the effectiveness of the proposed method. Results show that a direct correlation exists between the unreliability score of users and deceptive review identification. If the score exceeds 0.78 , then the reviewer is deemed to be a deceptive reviewer, and the reviews made are deceptive reviews. The proposed method provides a good prospect to identify deceptive reviews.
\end{abstract}

Keywords: Complex network, Network clustering, Community structure, Particle swarm optimization

\section{Introduction}

Most customers read online reviews when shopping on ecommerce sites. On the one hand, favorable reviews of a product give customers a good impression of it. On the other hand, bad reviews give a negative impression. In the ecompetitive platform, gaining favorable reviews is critical to the large-scale sales of goods. Therefore, many businesses are willing to pay a considerable amount of money to hire posters that will write favorable reviews for their products or negative reviews for their competitors.

Deceptive reviews do not necessarily mean that the quality of products is inferior. However, the appearance of deceptive reviews disturbs the environment of online shopping and increases the shopping risk of customers. Distinguishing deceptive reviews is difficult for customers without professional knowledge [1]. Generally, deceptive reviews are fraudulent, with a low rate of artificial perception. The number of deceptive reviews increases every day [2]. Moreover, deceptive reviews not only will bring unpleasant shopping experience to customers, but also do harm to the positive development of the entire ecommerce environment [3]. In addition, deceptive reviews will have a negative influence on academic research because academic studies are based on information such as product reviews. The adulteration caused by deceptive reviews makes the results of these academic studies less reliable.

On the basis of the above analysis, scholars have

*-mail address: 18846033037@163.com

ISSN: 1791-2377 @ 2019 Eastern Macedonia and Throce Institute of Technology. All rights reserved. doi:10.25103/iestr.121.23 conducted extensive research on the identification of deceptive reviews [4-6]. However, obtaining a genuine sample corpus of deceptive reviews is difficult. Most of the studies are in English, which do not conform to the Chinese grammatical features and structures. Moreover, the studies are based on spam reviews and the lack of pertinence. Therefore, how to enhance the accuracy and completeness of identifying deceptive reviews is an urgent problem.

Therefore, we propose a method of deceptive review identification that integrates sentiment analysis and the characteristics of reviewers. This proposed method utilizes a feature-weighted model to describe the emotional intensity of reviews and the importance of the characteristics of reviewers. In the process of deceptive review identification, the proposed method evaluates the reliability of each review to accurately identify deceptive reviews and improve the method based on traditional machine-learning algorithm.

\section{State of the art}

Currently, scholars have been doing notable research work on the identification of deceptive reviews. Kim et al. [7] proposed a framework approach based on deep semantics. In turn, this deep semantics is based on the gold standard data set of deceptive reviews published by Myle Ott et al. [8]. Their method is inefficient since it is based on third-party platforms. Their method is also hard to migrate to other platforms. Chen et al. [9] put forward a model for assessing emotional inclination based on the product attributes of reviews. This model was employed to identify the deceptive reviews of online products. The accuracy of the model 
depends on two important lexicons, namely, attribute and sentiment lexicons. The operation efficiency of the model is low, and the consumption time is long. Akoglu et al. [10] formed a network of reviewers and content of reviews. They used network effect to rate reviewers and the content of reviews. Content with low scores were marked as spam reviews. Some influence on the experimental results emerges because of the strong subjectivity of hand-annotated; Shao et al. [11] created a model of multilateral graph and proposed a method to identify deceptive reviewers. However, their method is not comprehensive in the analysis of the emotional characteristics of reviewers. A certain deviation in the manual annotation affected the evaluation of experimental results. Wang et al. [12] came up with an algorithm for mining suspected fraud. This algorithm was based on the similarity clustering of user informationsearching behavior. This behavior, in turn, comes from the online customer information searching behavior. The shortcomings of this algorithm lied on the discriminant threshold of "Click farm fraud cannot be automatically adjusted to identify suspected fraud." Fang et al. [13] brought forward a method for identifying deceptive reviews that integrates scoring evaluation consistency and multidimensional time series. Moreover, they constructed a model of deceptive review detection based on multi-dimensional time series. Multiple extracted deceptive review features were fused to construct a classifier of deceptive review detection without considering the dynamic comment situations. Zhao [14] proposed a systematic and low-cost “text-theme' double-level network method of deceptive review detection. The deceptive review identification method designed by the institute could not identify the reviews whose polarity was opposite to the genuine content of reviews.

The behavior of deceptive reviewers was considered by scholars all over the world to detect deceptive reviews. Dellarocas [15] first carried out cluster analysis on the comment behavior of reviewers. Further, Dellarocas classified deceptive reviews into two grades: overvaluation and undervaluation. The reviews published by low-credit reviewers were regarded as having no reference value, but it was too absolute to divide the reviews into two grades, which were not accurate most of the time. Kumar et al. [16], who conducted the study on the reviewers, analyzed the collective cheating behavior of several reviewers in accordance with their communication and interaction with the platform. They put forward the author fraud model, which inspired the research in this respect; however, its accuracy was not high enough. In their study, Liu et al. [17] categorized the deceptive reviews. A logistic regression model is proposed to identify deceptive reviews. Their method is only based on the assumption of reasonable classification; hence, it has some limitations. Lim et al. [18] started from the customer rating behavior. In addition, they built the model manually according to experience, set the weight of the various behavioral characteristics of reviewers, and found the source through the weight. This method is only applicable to those websites with the customer rating function. Qiu et al. [19] selected reviewers according to a single index. The method of the integrated selection of five indicators determines the number of reviewers who are most and least likely to become spam reviewers and marks them manually. A supervised linear regression model was designed on the basis of the results of markers. Given the strong subjectivity of artificial markers, the results may be affected. Xie et al. [20] applied the detection method of multiple time scale, taking advantage of characteristics such as the release time of reviews and the historical release records of reviewers. By analyzing the time window of the centralized release of deceptive reviews in time sequence, a single review in the window is regarded as a deceptive review. However, treating a single review as a deceptive review is just a probability event, which is also unstable. Xing et al. [21] presented a method of deceptive review identification based on a Markov logic network, which can extract the characteristics of behavior and text of reviewers and form a first-order formula. The limitation of this method is that with the increase of data, the formation of the Markov network becomes more time-consuming and complex, which is not suitable for large-scale data sets. $\mathrm{Yu}$ et al. [22] proposed a model in 2016, which can identify deceptive review customers and sellers who have swiped points. The disadvantage of this model is that it does not consider the structural, grammatical, and semantic problems of the text itself, and many algorithms lack theoretical support. Hernandez Fusilier et al. [23] analyzed the role of opinion polarity in the detection. Moreover, they corroborated that negative deceptive reviews were more difficult to detect than positive deceptive reviews. This finding was also related to the reviewers. Specific conclusions were not given and must still be studied.

The above methods of deceptive review identification are mainly based on the content of reviews or the behavior of the reviewers. Given the increasing number of deceptive reviews, performing only one method of identification is not ideal. When analyzing from the perspective of the review content or the behavior of the reviewers, taking the other side to supplement the identification should provide more accuracy. Therefore, the present study considered the characteristics of the review content and the reviewers' behavior and proposed a method of deceptive review identification by integrating sentiment analysis and the characteristics of reviewers. This proposed method used the feature-weighted model to evaluate the reliability of the reviews to identify deceptive reviews accurately.

The rest of this study is organized as follows. Section 3 establishes the method integrating sentiment analysis and the characteristics of reviewers. Deceptive reviewers can be identified by calculating the unreliable fraction of reviewers; therefore, deceptive reviews can be identified. Section 4 constructs the data set by means of crowd-sourcing, and the accuracy and performance of the deceptive review identification method created are verified by the comparison experiment. Section 5 concludes the study.

\section{Methodology}

\subsection{Deceptive review identification based on sentimental analysis}

We analyze the sentiment of the text through text preprocessing, emotion level annotation, and weight analysis. The authenticity of the reviews is tested by the emotion intensity of the reviews.

\subsubsection{Text preprocessing}

The text is pre-processed first to facilitate subsequent experiments, mainly including the following contents:

(1) Invalid values must be removed. For example, in the raw data, the system provides the default value as "positive 
Xinyu Gao, Shi Li, Yiyin Zhu, Yiting Nan, Zihan Jian and Hongyu Tang/

Journal of Engineering Science and Technology Review 12 (1) (2019) 196 - 202

opinion." These values are not helpful for evaluation; hence, they are filtered out.

(2) Non-text characters must be removed. These are special characters, punctuation marks, and numbers. These non-text characters are meaningless for text mining; hence, they must be removed.

(3) Stop words must be removed. Some meaningless words frequently appear in the text documents. For instance, in the text documents, many words emerge, such as "and," "with," and "proviso," which are defined as stop words. They do not contribute to the content of the text and hinder the understanding of the content of the text; hence, they must be removed from text analysis. The present study adopts the stop-word list of the Harbin Institute of Technology to remove the stop-words in the text.

\subsubsection{Sentimental analysis}

The sentiments of customers are usually expressed through the emotional words in the reviews. For the same evaluated object, the emotional inclination and degree of expression vary from person to person. For instance, commenting on the same item of clothing, ordinary customers will use positive words to express their sentiments. Meanwhile, the deceptive favorable reviews written by hired professional writers tend to use more compliments than ordinary customers, with a stronger sentiment opinion and some exaggerated languages. The use of emotional words in reviews can show and strengthen the sentiment polarity of reviews [24] (Table 1).

Table 1. Differences of Sentiment Polarity between a Genuine Review and a Deceptive Review

\begin{tabular}{|c|c|c|c|}
\hline Sequence & Review Instance & $\begin{array}{l}\text { Emotional } \\
\text { Intensity }\end{array}$ & $\begin{array}{l}\text { Review } \\
\text { Type }\end{array}$ \\
\hline 1 & $\begin{array}{l}\text { The clothes are okay, } \\
\text { anyway, it is worth } \\
\text { every penny. However, } \\
\text { the delivery is too slow. } \\
\text { The delivery speed is } \\
\text { very fast, the consumer } \\
\text { service is very friendly, } \\
\text { all questions were } \\
\text { answered, and I am } \\
\text { very happy with this } \\
\text { purchase. The clothes } \\
\text { were received and the }\end{array}$ & Weak & $\begin{array}{l}\text { Genuine } \\
\text { Reviews }\end{array}$ \\
\hline 2 & $\begin{array}{l}\text { thickness is unexpected. } \\
\text { There is no color } \\
\text { difference. It feels } \\
\text { super good when } \\
\text { touched outside. Indeed } \\
\text { a good choice. Very } \\
\text { good store, very good } \\
\text { quality, and I am very } \\
\text { satisfied. }\end{array}$ & Strong & Reviews \\
\hline
\end{tabular}

The pre-processed data set is put into the process of word segmentation and calculation of word frequency by matching with the Hownet and simplified Chinese emotion lexicons of Taiwan. The outputs are positive and negative word frequency lexicons. The present study must describe the sentimental orientation of the text and the level of sentimental orientation to express the degree of positivity or negativity. Therefore, all the emotion words in the lexicon are sorted by word frequency. The top 50 words with the highest frequency were rated and assigned values. Positive words were positive values, while negative words were negative values.

We crowd-source 359 deceptive and 375 genuine reviews to form the training set. The emotional terms (number) in the training set are counted as $\left(N_{w}\right)$.
The emotion intensity of a review is reflected by the emotional term, intensity. Thus, the weight of each review $\left(W_{t}\right)$ in the training set should be equal to the sum of the weight of positive words $\left(W_{p}\right)$ and negative words $\left(W_{n}\right)$ in the review:

$W_{t}=\sum W_{p}+\sum W_{n}$

Table 2. Experimental Result of Genuine versus Deceptive Review Emotional Intensity

\begin{tabular}{c|c}
\hline $\begin{array}{c}\text { Deceptive Review } \\
\text { Emotional Intensity }\end{array}$ & $\begin{array}{c}\text { Genuine Review Emotional } \\
\text { Intensity }\end{array}$ \\
\hline 0.9592 & 0.4828 \\
0.9502 & 0.7140 \\
0.8647 & 0.7637 \\
0.9305 & 0.3762 \\
0.6321 & 0.7472 \\
0.9225 & 0.7855 \\
0.8089 & 0.7568 \\
0.8513 & 0.8169 \\
0.7928 & 0.6848 \\
0.8177 & 0.4467 \\
0.8609 & 0.6959 \\
0.8464 & 0.7644 \\
0.8348 & 0.6985 \\
0.9301 & 0.7659 \\
0.9059 & 0.5331 \\
0.8528 & 0.7530 \\
0.9225 & 0.6873 \\
0.9244 & 0.6075 \\
0.8173 & 0.6694 \\
\hline
\end{tabular}

Given that the weight obtained here is the sum of the weights of all emotion terms in the review text, the present study considers the absolute value of this weight as the emotional intensity value of the reviews. In addition, to normalize the emotional intensity value of the review to $[0,1]$ scope while preventing the influence of the overweight of the positive words or the underweight of the negative words on the emotional intensity of the review, inspired by Wang et al. [25], the present study adopts the following curve function to smooth $\left(W_{t}\right)$ to calculate the emotional intensity of the review $(E)$ :

$$
E_{t}=1-\frac{1}{e^{\left|W_{t}\right| / N_{w}}}
$$

The larger the value of $E_{t}$, the stronger the emotional intensity. Through the training and tuning of the training data set, we get the following results in Table 2 .

\subsection{Deceptive review detection based on characteristics of reviewers}

The deceptive review identification based on the characteristics of reviewers is analyzed from three aspects to improve the accuracy of deceptive review identification.

\subsubsection{Content similarity}

Copying is a common method used by the publishers of deceptive reviews. Compared with that of fabricating a new review, the time cost of copying the original review of a 
Xinyu Gao, Shi Li, Yiyin Zhu, Yiting Nan, Zihan Jian and Hongyu Tang/

Journal of Engineering Science and Technology Review 12 (1) (2019) 196 - 202

similar product is significantly lower. Mukher Jee et al. [26] analyzed the Yelp data set to show that more than $70 \%$ of deceptive review publishers posted reviews with similarities greater than 0.3 , while genuine reviewers posted reviews with similarities below 0.18 . For the same reviewer, a content similarity calculation helps in capturing the behavioral characteristics of reviewers.

The Chinese text classification feature and feature weighting method [27] are employed to do some basic data cleaning operations to detect duplicate reviews. First, a language model was built for all reviews. Thereafter, a similarity value $m\left(p_{i}, p_{j}\right)$ is calculated for two reviews $\mathrm{p}_{i}$ and $\mathrm{p}_{\mathrm{j}}$. The larger the similarity value, the more likely it is that the two reviews are duplicate reviews. Through analysis and calculation, four main types of repeated reviews emerge:

1) Different reviewers made the same reviews on the same product.

2) Different reviewers made the same reviews on different products.

3) Same reviewers made the same reviews on different products.

4) Same reviewers made the same reviews on the same product.

\subsubsection{Maximum number of reviews posted on the day}

Submitting several reviews in a single day is an anomalous behavior [28]. Mukher Jee et al. [26] found in an analysis of Yelp data set that approximately $75 \%$ of deceptive reviewers posted more than five reviews per day, whereas more than $90 \%$ of genuine reviewers had no more than three reviews per day.

The present study counts the number of reviews published by the reviewer in a certain period. The number of reviews published by the deceptive reviewer in a certain period is commonly very large; hence, the number of reviews published is used as a one-dimensional feature. If the reviewer has too many reviews in a certain period, then the reviewer is a deceptive reviewer, and the reviews published are also deceptive.

\subsubsection{Extreme rating behavior}

We treat the highest or the lowest score on product rating as an extreme rating with the possibility that the reviewer deliberately praises or disparages the product. Rating a product with one star and five stars in a five-star rating system is an extreme rating behavior.

\subsection{Reviewer unreliability score}

The present study combines sentiment analysis and the characteristics of reviewers to calculate reviewer unreliability scores.

Table. 3. Symbol Definition

\begin{tabular}{l|l}
\hline Symbol & Definition \\
\hline$r$ & Reviewer \\
$n_{c}$ & Review Text \\
$E_{i}$ & Set of Review Text \\
$\overline{E(r)}$ & Emotional Intensity Value of the Review \\
$d_{i}$ & Emotional Intensity Value of the Reviewer \\
$D_{c}$ & Number of Reviews Published Per Unit of Time \\
$m\left(p_{i}, p_{j}\right)$ & Review Number of Reviews in a Unit of Time \\
& Review $p_{i} \& p_{j}$ 's Text Similarity
\end{tabular}
$f r(c)$
$f i(c)$
$c i(r)$
$\mathrm{cm}(r)$
$c e(r)$
$U S(r)$

1)The emotional intensity for all the reviews of a reviewer must be calculated. The mean of the emotional intensity is taken as the value of the emotional intensity of the reviewer:

$$
\overline{E(r)}=\frac{\left|\sum E_{i}\right|}{\left|n_{c}\right|}, i \in n_{c}
$$

2)All the review dates of the reviewers must be counted, the proportion of each date in all dates calculated, and the maximum value taken as the largest daily publishing review index of a reviewer:

$$
c i(r)=\max x \mid x=\frac{\left|d_{i}\right|}{\left|n_{c}\right|}, d_{i} \in D_{c}
$$

3)All the review texts of a reviewer in pairs must be compared, and the maximum value must be taken as the text similarity of the reviewers:

$$
c m(r)=\max \left\{s \mid s=m\left(p_{i}, p_{j}\right), i, j \in n_{c}\right\}
$$

4)All the star ratings of a reviewer must be counted, the proportion of four and five stars in all ratings calculated, and the proportion taken as a reviewer's extreme rating index.

$$
c e(r)=\frac{|f r(c)+f i(c)|}{\left|n_{c}\right|}
$$

The unreliability score of the reviewers is determined by the emotional intensity value, the largest daily publishing review index, the text similarity, and the extreme rating index which are all positively correlated. By assigning weights, a formula for unreliability scores can be obtained. Therefore, the calculation method for the unreliability score of the reviewers defined in the present study is as follows:

$U S(r)=\alpha \overline{E(r)}+\beta c i(r)+\chi c m(r)+\varepsilon c e(r)$

In the present study, $\alpha$ must be set to $0.4, \beta$ to $0.1, \chi$ to 0.3 , and $\varepsilon$ to 0.2 . The formula shows that when the review texts have high emotional intensity value, when several reviews per unit time emerge, when a high text

similarity occurs, and when many extreme reviews are available, the unreliability score of the reviewer will increase accordingly.

Through the experiment on the training data set, we have the following experimental results as shown in Table 4 .

The experimental results contend that the unreliability scores of deceptive reviewers are generally higher than 0.78 , while the unreliability scores of genuine reviewers are generally lower than this value. Therefore, the reviewers with unreliability scores greater than 0.78 are included in the 
Xinyu Gao, Shi Li, Yiyin Zhu, Yiting Nan, Zihan Jian and Hongyu Tang/

Journal of Engineering Science and Technology Review 12 (1) (2019) 196 - 202

candidate set of the deceptive reviewers. The reviews published are included in the collection of deceptive reviews.

\subsection{Detection steps}

The deceptive review identification method adopted considers the intensity of emotions of the review texts and the relationship between the reviewer and the feature of its reviews.

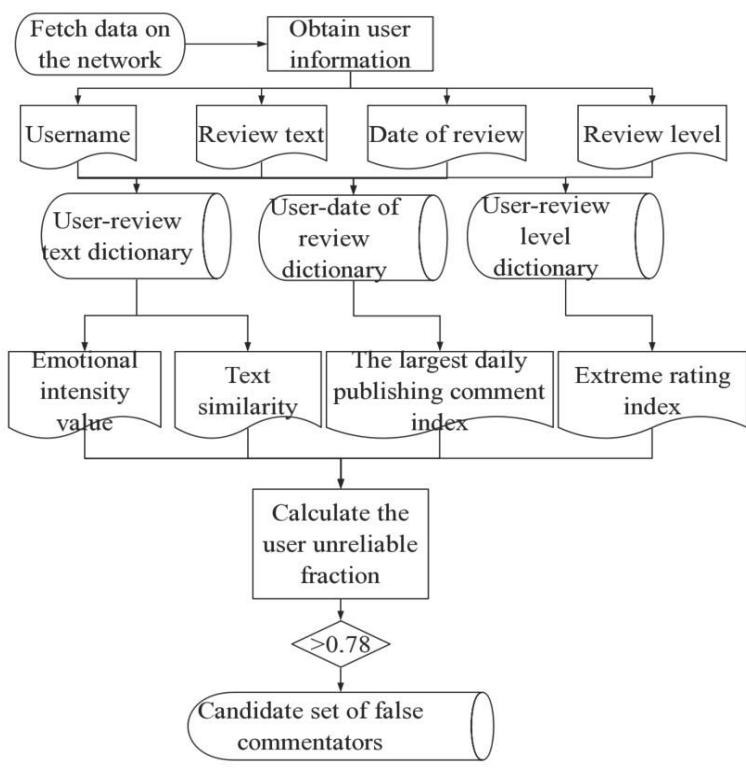

Fig. 1. Detection Steps

Specific steps are as follows:

Input: customer, review date, review text, review star Output: deceptive reviewer candidate set

Step 1) customer-review date lexicon, customer-review text lexicon, and customer-review star lexicon must be created.

Step 2) The emotional intensity value of each review must be calculated according to Equations (1) and (2).

Step 3) The emotional intensity value of the reviewers must be calculated according to Equation (3).

Step 4) The maximum daily release index of the reviewers must be calculated according to Equation (4).

Step 5) The text similarity of the reviewers must be calculated according to Equation (5).

Step 6) The extreme rating index of reviews must be calculated according to Equation (6).

Step 7) The final unreliability score of the reviewers must be calculated according to Equation (7).

Step 8) A reviewer with an unreliability score greater than 0.78 must be incorporated into the deceptive reviewer candidate set and the reviews made into the deceptive review set.

Table 4. Experimental Result of Genuine versus Deceptive Reviewers' Unreliability Scores

\begin{tabular}{|c|c|}
\hline $\begin{array}{ll}\text { Deceptive } & \text { Reviewers' } \\
\text { Unreliability Scores } & \\
\end{array}$ & $\begin{array}{ll}\text { Genuine } & \text { Reviewers' } \\
\text { Unreliability Scores } & \end{array}$ \\
\hline 0.7748 & 0.7489 \\
\hline 0.7928 & 0.7027 \\
\hline 0.8177 & 0.6544 \\
\hline 0.8609 & 0.7744 \\
\hline 0.9306 & 0.6750 \\
\hline 0.8000 & 0.6780 \\
\hline 0.8649 & 0.7070 \\
\hline 0.7873 & 0.8297 \\
\hline 0.7809 & 0.7016 \\
\hline 0.7602 & 0.7728 \\
\hline 0.9055 & 0.7722 \\
\hline
\end{tabular}

\begin{tabular}{c|c}
\hline 0.8348 & 0.7261 \\
\hline 0.9301 & 0.7492 \\
\hline 0.8330 & 0.7574 \\
\hline 0.9382 & 0.8114 \\
\hline 0.8602 & 0.7002 \\
\hline 0.8259 & 0.6864 \\
\hline 0.8248 & 0.7682 \\
\hline 0.8214 & 0.6389 \\
\hline 0.7989 & 0.6844
\end{tabular}

\section{Result analysis and discussion}

\subsection{Construction of the data set}

The data set from the review website and the crowd-sourcing method is divided into experiment and training sets, respectively.

\subsubsection{Crowd-sourcing data set}

Ott et al. [29] used the crowd-sourcing platform to construct a gold standard data set that can be used for identification for deceptive reviews. The labeled data sets built by the crowdsourcing platform were constructed manually, and no misjudgment emerged. The present study borrows the rules used by Myle Ott et al. [29] to create genuine and deceptive review data sets as the training set of the model.

\subsubsection{Data Set of Review Website}

Mayzlin [30] and Ott et al. [31] demonstrated that reviews on the Amazon Chinese online shopping platform are very representative. The present study gathers review data of Amazon while many members annotate the review data at the same time. The mode of the annotated result is taken as the final annotation. A part of the annotated review data is then extracted as the experimental test set. In view of the protection of shops of Amazon, the present study does not display any information about the store. The review data of customers in Amazon Chinese belongs to dynamic storage. The present study uses Python crawler technology to obtain JavaScript Object Notation (JSON) for storing product reviews and review data by parsing JSON.

The data set contains the following types of information: customer name, customer ID, review text, review posting time, and star rating of customer reviews. The related information of the two has certain auxiliary functions for the judgment of the falseness of the reviews.

The crawled review data are taken as the sample to be labeled. The sample is manually labeled "Genuine Reviewer" or "Deceptive Reviewer." For each sample to be labeled, the mode of the labeled result is taken. The annotation sample is expanded into the genuine reviewers and the deceptive reviewer's data set. A total of 40 genuine reviewers and 40 deceptive reviewers [32] are randomly selected from the data sets as experimental sets. Table 5 shows the data sets.

Table 5. Experimental Data Information

\begin{tabular}{c|c|c|c}
\hline Experimental Data & Deceptive & Genuine & Total \\
\hline Reviewer & 112 & 620 & 732 \\
\hline Review & 633 & 3991 & 4624 \\
\hline
\end{tabular}

\subsection{Experimental Design}

For the verification of the validity and accuracy of the identification method integrating sentiment analysis and characteristics of reviewers, the experiment is designed as two comparative experiments: 
Xinyu Gao, Shi Li, Yiyin Zhu, Yiting Nan, Zihan Jian and Hongyu Tang/

Journal of Engineering Science and Technology Review 12 (1) (2019) 196 - 202

1) Comparison and combination of the identification of sentimental orientation features and the identification of text reviewers

2) Comparison of the experimental results of different methods.

Accuracy, recall, and F-score are considered evaluation indicators in the experiment.

\subsection{Results and analysis of experiment}

For the analysis of the identification effect of deceptive reviews integrating sentiment analysis and characteristics of reviewers, the experiment is divided into three categories:

1) Identification of sentiment analysis refers to the analysis of text from the perspective of emotional polarity, characterized by emotional intensity. This type is used as T1.

2) Identification of the characteristics of reviewers, which combines the content similarity of reviewers, the maximum number of the reviews published, and the extreme rating behavior. This type is used as $\mathrm{T} 2$.

3) Identification integrating sentiment analysis and characteristics of reviewers, that is to identify all the features by combining the characteristics of sentimental orientation and reviewers. This type acts as $\mathrm{T} 3$.

Table 6 exhibits the results of each type of experiment. Table 6 shows that Accuracy A and F1 values of type T3 are 0.92, 0.17 higher than T1. Accuracy A, the recall R, and

Table 6. Each Type of Experimental Results

\begin{tabular}{l|l|l|l}
\hline Data & T1 & T2 & T3 \\
\hline Accuracy & 0.64 & 0.91 & 0.93 \\
Recall & 0.90 & 0.86 & 0.90 \\
F1 & 0.75 & 0.88 & 0.92 \\
\hline
\end{tabular}

F1 values of the type T3 are $0.02,0.04,0.04$ higher than T2. Calculated from the table, M3 comprehensive performance has $23 \%$ improvement over $\mathrm{T} 1$ and a $5 \%$ improvement over $\mathrm{T} 2$. The experimental results confirm that type $\mathrm{T} 3$ has higher accuracy and better recall ability. The experimental results prove the effectiveness of the identification of deceptive reviews integrating sentiment analysis and characteristics of reviewers and improvement of the performance.

For the further verification of the validity of the proposed method and the effect of deceptive review identification, the method presented is compared with the methods proposed in some existing studies. (1) T1: Xing Juanjuan et al. [21] Deceptive Reviews identification Method Based on a Markov Logic Network. (2) T2: Pi Qi et al. [33] Deceptive Review Identification Based on Deep Learning. (3) T3: The present study proposes a combination of emotional sentiment feature identification and text reviewer combined identification method. Graph 1 depicts the effect of each method on the identification of deceptive reviews:

Fig. 2 shows that the identification method of integrating sentiment analysis and the characteristics of reviewers has been further improved compared with the two existing methods, with higher accuracy, better recall and better F1 value. This comparison indicates that the proposed method has higher validity and accuracy and better effect of deceptive review identification.

\section{Conclusion}

To improve the performance of the deceptive review identification and accurately identify deceptive reviews, the present study considered the difference in sentiment polarity between genuine and deceptive reviews based on integrating sentiment analysis and the characteristics of reviewers. The proposed method analyzed the emotional characteristics of deceptive reviews and used the feature-weighted model to analyze the importance of four aspects to evaluate the reliability of the reviewers. The deceptive reviewers were identified by their unreliability scores. Accordingly, a deceptive reviewer candidate set was obtained. The following conclusions could be drawn:

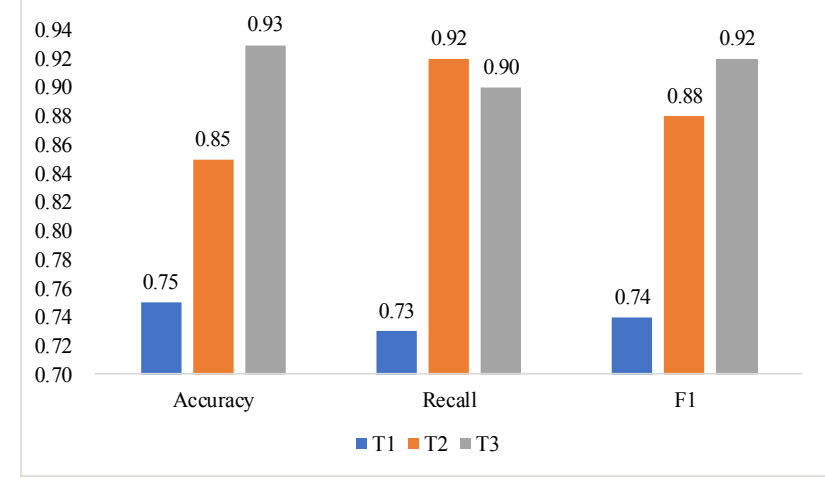

Fig. 2. Deceptive Reviews Recognition Effect Of Different Methods

(1) The higher the emotional intensity value of the review, the higher the probability that it is a deceptive review. The emotional intensity value exceeding 0.8 is generally considered a deceptive review.

(2) The text similarity of the reviews, the largest daily publishing review index, and the extreme rating index are positively correlated with the likelihood that the review is deceptive.

(3) The higher the unreliable scores of the reviewer, the lower the authenticity of the review. Reviewers with unreliability scores of above 0.78 are commonly deceptive reviewers.

In sum, the present study combines sentiment analysis with reviewer behavior analysis and then proposes a deceptive review identification method integrating sentiment analysis and the characteristics of reviewers. The proposed method improves the accuracy of deceptive review identification and the performance of deceptive review identification. The present study plays a certain reference role in the subsequent research on deceptive review identification integrating sentiment analysis and the characteristics of reviewers. As for the certain deviation in manual marking, we propose that in future studies, a machine-learning method be adopted to mark the data set, which will be combined with the proposed method so that deceptive reviews can be recognized accurately.

\section{Acknowledgements}

The study was supported by the National Undergraduate Training Programs for Innovation and Entrepreneurship (Grant No.201810225174) and the Fundamental Research Funds for the Central Universities (Grant No. 2572015CB33).

This is an Open Access article distributed under the terms of the Creative Commons Attribution License

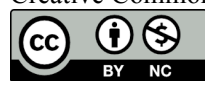




\section{References}

1. Han Yutan, "Deceptive Reviews identification Model Based on Neural Networks". Scientific and Technological Innovation, (06),2018, pp.78-79.

2. Song Haixia, Yan Xin, Yu Zhengtao, "Deceptive Reviews detection Based on Adaptive Clustering". Journal of Nanjing University (Natural Science),49 (4), 2013, pp. 433-438.

3. Zhang Liyi, Liu Chang, "Research on False Transaction identification Based on Deep Belief Network and Fuzzy Set". Data Analysis and Knowledge Discovery, 266(1),2016, pp. 32-39.

4. Ren Yafeng, Yin Lan, Ji Donghong, "Deceptive Reviews identification Based on Language Structure and Sentiment Polarity". Journal of Frontiers of Computer Science and Technology, 8(03),2014, pp. 313-320.

5. Chen C. C., Tseng Y., "Quality Evaluation of Product Reviews Using an Information Quality Framework". Decision Support Systems, 50(4),2011, pp. 755-768.

6. Zhang Wenyu, Yue Kun, Zhang Binbin, "Detection of False Commentators in E-Commerce Based on D-S Evidence Theory". Journal of Chinese Computer Systems, 39(11), 2018, pp. 24282435.

7. Kim S, Chang H, Lee S, et al., "Deep semantic frame-based deceptive opinion spam analysis" In: Proceedings of the 24th International on Conference on Information and Knowledge Management, Melbourne, Australia: ACM,2015, pp.1131-1140.

8. Ott M, Choi Y, Cardie C, et al., "Finding deceptive opinion spam by any stretch of the imagination" In: Proceedings of the 49th Annual Meeting of the Association for Computational Linguistics: Human Language Technologies-Volume, Stroudsburg, USA: Association for Computational Linguistics, 2011, pp.309-319.

9. Chen Yanfang, Li Zhiyu, "Research on Deceptive Reviews identification Based on Evaluation of Emotional Inclination of Comment Product Attributes". Data Analysis and Knowledge Discovery, (09), 2014, pp.81-90.

10. Akoglu L, Chandy R, Faloutsos C, "Opinion Fraud Detection in Online Reviews by Network Effects". ICWSM, (13), 2013, pp. 211.

11. Shao Zhufeng, Ji Donghong, "False Commentators identification Based on Emotional Characteristics and Customer Relation". Computer Applications and Software, 33(05),2016, pp. 158$161+172$.

12. Wang Zhongqun, Yue Yuan, "Identification of Conspiracy Sales Volume Fraud Based on Template Customer Information Search Behavior and Statistical Analysis". Data Analysis and Knowledge Discovery, 264(11),2015, pp. 41-50.

13. Fang Youli, Wang Hong, "Deceptive Reviews identification Based on Score - evaluation consistency and Multidimensional time series”. Journal of Chinese Computer Systems, 39(09),2018, pp. 2044-2049.

14. Zhao Yan, "Research on Network Deceptive Reviews Detection Based on 'Text-Theme' Bi-Level Analysis: Take an E-Commerce Website as an Example". Digital Library Forum, (09),2015, pp. 5360 .

15. Dellarocas C., "Mechanisms for coping with unfair ratings and discriminatory behavior in online reputation reporting systems". In: Proceedings of the twenty first international conference on Information systems, Queensland, Australia: Association for Information Systems, 2000, pp. 520-525.

16. Mukherjee A, Kumar A, Liu B, et al., "Spotting opinion spammers using behavioral footprints". In: Proceedings of the 19th ACM SIGKDD international conference on Knowledge discovery and data mining, New York, USA:ACM, 2013, pp. 632 -640
17. Jindal N., Liu B., "Review spam detection". In: Proceedings of the 16th international conference on World Wide Web, New York, USA:ACM, 2007, pp.1189-1190.

18. Lim E.P., Nguyen V. A., Jindal N., et al., "Detecting product review spammers using rating behaviors". In: Proceedings of the 19th International Conference on Information and Knowledge Management, New York, USA:ACM, 2010, pp.939-948.

19. Qiu Yunfei, Wang Jiankun, Shao Liangshan, Liu Dayou, "Product Waste Commentators Detection Research Based on Customer Behavior". Computer Engineering,38(11), 2012, pp. 254-257+261.

20. Xie S., Wang G., Lin S., et al., "Review spam detection via temporal pattern discovery". Proceedings of the International Conference on Knowledge Discovery and Data Mining, Beijing, China,2012, pp.823-831

21. Xing Juanjuan, "Deceptive Reviews identification Method Based on Markov Logic Network". Journal of Chinese Information Processing, 30(05),2016, pp. 94-100.

22. Yu Chuanming, Feng Bolin, Zuo Yuheng, Chen Baiyun, An Lu, "Research on Deceptive Reviews identification Based on Individual-Group-Merchant Relationship Model". Acta Scientiarum Naturalism Universitatis Pekinensis,53(02), 2017, pp. 262-272.

23. Fusilier D H, Montes-y-Gómez M, Rosso P, et al., "Detecting positive and negative deceptive opinions using PU-learning". Information processing \& management, 51(4), 2015, pp. 433-443.

24. Li J., Ott M., Cardie C., et al., "Towards a general rule for identifying deceptive Opinion spam". In: Proceedings of the Meeting of the Association for Computational Linguistics, Baltimore, USA: Association for Information Systems, 2014, pp.1566-1576.

25. Wang G., Xie S., Liu B. and Yu P., "Review Graph Based Online Store Review Spammer Detection", In: Proceedings of the 11th International Conference on Data Mining, Vancouver, BC: IEEE, 2011, pp. 1242-1247.

26. Mukherjee A., Venkataraman V., Liu B., Glance N., "What yelp fake review filter might be doing" Proceedings of the International AAAI Conference on Web and Social Media. Washington, USA,2013, pp.409-418

27. Wang Yong, "Research on Chinese Text Classification Feature Selection and Feature Weighting Method". Master thesis of Chongqing University, 2012.

28. Li Luyang, Qin Bing, Liu Ting, "The Research Summary of deceptive reviews detection". Chinese Journal of Computers, 41(04),2018, pp. 946-968.

29. Jiwei Li, Myle Ott, Claire Cardie, Eduard H. Hovy, "Towards a general rule for identifying deceptive opinion spam". In: Proceedings of the 52nd Annual Meeting of the Association for Computational Linguistics, Baltimore, USA: Association for Computational Linguistics,2014, pp. 1566-1576.

30. Chevalier J.A., \&Mayzlin D., "The effect of word of mouth online: online book reviews". Journal of Marketing Research, 43(3),2006, pp.345-35

31. Myle Ott, Yejin Choi, Claire Cardie, "Finding Deceptive Opinion Spam by Any Stretch of the Imagination". In: Proceedings of the 49th Annual Meeting of the Association for Computational Linguistics: Human Language Technologies, Stroudsburg, USA: Association for Computational Linguistics, 2011, pp.309-319.

32. Ren Yafeng, Ji Donghong, Zhang Hongbin, "Research on Deceptive Reviews identification Based on Learning Algorithm of Positive and Unlabeled". Journal of Computer Research and Development, 52(3), 2015, pp. 639-648.

33. Pi Qi, Wang Wenjie, Yang Fei, Zhao Yao, "Deceptive Reviews identification Based on Deep Learning", Journal of Network New Media, 5(06) ,2016, pp.30-33. 